\title{
The role of cytokinins in clubroot disease
}

\author{
Robert Malinowski • Ondřej Novák • \\ M. Hossein Borhan • Lukáš Spíchal • \\ Miroslav Strnad • Stephen A. Rolfe
}

Accepted: 18 December 2015 / Published online: 14 January 2016

(C) The Author(s) 2016. This article is published with open access at Springerlink.com

\begin{abstract}
Clubroot (Plasmodiophora brassicae) is a pathogen of Brassicaceae that causes significant reductions in yield as a consequence of gall formation in the root and hypocotyl of infected plants. The pathogen hijacks host vascular cambium development, and cytokinins are implicated in this process. This paper uses transcriptomics and metabolomics to investigate changes in cytokinin metabolism during gall formation of clubrootinfected Arabidopsis thaliana. RNASeq analysis of infected tissue showed that host cytokinin metabolism was strongly down-regulated both at the onset and late stages of gall formation. Expression of host genes associated
\end{abstract}

Electronic supplementary material The online version of this article (doi:10.1007/s10658-015-0845-y) contains supplementary material, which is available to authorized users.

\section{R. Malinowski}

Department of Integrative Plant Biology, Institute of Plant Genetics of the Polish Academy of Sciences, ul. Strzeszynska 34, 60-479 Poznan, Poland

O. Novák · L. Spíchal · M. Strnad

Laboratory of Growth Regulators, Centre of the Region

Haná for Biotechnological and Agricultural Research,

Institute of Experimental Botany ASCR and Palacký University,

Olomouc, Czech Republic

M. H. Borhan

Saskatoon Research Centre, Agriculture and Agri-Food Canada, Saskatoon, SK S7N 0X2, Canada

S. A. Rolfe $(\bowtie)$

Department of Animal and Plant Sciences, University of Sheffield, Sheffield S10 2TN, UK

e-mail: s.rolfe@sheffield.ac.uk with cytokinin biosynthesis, signalling, degradation and conjugation was strongly repressed. Analysis of cytokinin precursors, active components and conjugates by microanalytical techniques was consistent with these transcriptional responses. Two isopentenyltransferase genes associated with cytokinin biosynthesis are present in the P. brassicae genome and are expressed throughout gall formation. The impact of pathogen-derived cytokinins on the total cytokinin content of infected tissue and host gene expression was minimal in wild type plants. However, infection of ipt $1 ; 3 ; 5 ; 7$ mutants that are severely restricted in their ability to synthesise active cytokinins led to an increase in expression of host cytokininresponsive genes. We interpret these results as indicating that $P$. brassicae can synthesise small amounts of cytokinin, but this has little impact on the host plant as the ipt 1;3;5;7 phenotype is not rescued. Intriguingly, plasmodial development was slowed and spore viability reduced in these mutants indicating a potential role for cytokinins in plasmodial development.

Keywords Clubroot Plasmodiophora brassicae . Cytokinins $\cdot$ Transcriptomics $\cdot$ Metabolomics

\section{Introduction}

Clubroot is an economically important pathogen of Brassicas leading to reductions in yield and, in cases of severe infection, death of the host plant (Dixon 2009). It has a complex lifecycle which makes it particularly difficult to eradicate from infested land. Long-lived 
spores in the soil germinate to release primary zoospores that infect root hair cells. These undergo a series of divisions eventually generating secondary zoospores that penetrate the cortex of the root. At this stage the gall characteristic of clubroot disease develops. Extensive hypertrophy and hyperplasia occurs disrupting the host tissues, interfering with water relationships and generating a strong sink that reduces yield (Kageyama and Asano 2009). Clubroot also infects the model plant Arabidopsis thaliana (Mithen and Magrath 1992) and recent studies have shown that gall formation in this species occurs as a result of the pathogen manipulating the host developmental pathways associated with secondary thickening (Malinowski et al. 2012).

Gall formation is thought to involve perturbations in phytohormone homeostasis, particularly of auxin and cytokinin and more recently brassinosteroids, contributing to the altered morphogenic processes within the root and hypocotyl (Ludwig-Müller et al. 2009; Schuller et al. 2014). Studies of P. brassicae-infected Arabidopsis have shown that in the early stages of infection, elevated cytokinins are associated with increased cell division. This is thought to occur as a result of a complex interaction between host and pathogen metabolism and signalling. At later stages of gall formation the expression of host cytokinin biosynthetic genes is repressed, but so too is the expression of host cytokinin oxidases and dehydrogenases (Devos et al. 2006; Siemens et al. 2006). However, the measurement of many CK species, isomers, conjugates and degradation products is technically difficult and many components are either not resolved (Devos et al. 2006) or high numbers of biological replicates are required (Devos et al. 2005). The pathogen may also have the capability to synthesise cytokinins from nucleotide precursors (Müller and Hilgenberg 1986). Interfering with the balance of CK synthesis and degradation has the potential to impact disease progression. For example, plants constitutively overexpressing cytokinin oxidase show reduced gall formation (Siemens et al. 2006). Cytokinins and auxins act in concert to regulate tissue development. In later stages of infection, alterations in the concentrations and transport of auxin, and auxin-related metabolites such as indoleglucosinolates, are thought to be important although published data are contradictory (Devos et al. 2005; Ludwig-Müller et al. 2009). It has been proposed that P. brassicae plasmodia act as a sink for IAA and that host auxin biosynthetic reactions are stimulated. Elevated auxins are thought to lead to increases in cell wall extensibility associated with cell expansion (Devos et al. 2005; Ludwig-Müller et al. 2009).

In this report we have combined a transcriptomic approach using RNASeq and quantitative analysis of the CK content of control and clubroot-infected Arabidopsis to provide a comprehensive overview of the CK metabolism of developing galls. Previous studies using microarray analysis of host gene expression have been crucial in developing our understanding of this plant-pathogen interaction (Siemens et al. 2006; Agarwal et al. 2011; Schuller et al. 2014) but RNASeq provides greater coverage of host gene expression and greater sensitivity at very high or low expression. The recent completion of the P. brassicae genome (Schwelm et al. 2015 and studies in our laboratories) also allows the transcriptional response of both the host and pathogen to be studied simultaneously. The development of ultrasensitive methods for the quantification of CKs and their metabolites in milligram amounts of tissue has allowed us to develop a complete $\mathrm{CK}$ and auxin profile of control and infected tissue. The completion of the $P$. brassicae genome has allowed the identification of pathogen CK biosynthetic genes and we have examined their expression in infected tissue. We have also exploited the molecular tools available in Arabidopsis to examine the relative contributions of the host and pathogen transcriptome to $\mathrm{CK}$ biosynthesis.

\section{Materials and methods}

\section{Biological material}

Arabidopsis thaliana Col-0 was used for all experiments. The P. brassicae isolate is defined as European Clubroot Differential set $16 / 2 / 12$ and is described in Devos et al. (2005). Plants were inoculated with $1 \mathrm{ml}$ of $10^{6}$ spores $\mathrm{ml}^{-1}$. Plant growth, inoculations, RNA preparation, GUS staining and microscopy were performed as described in Malinowski et al. (2012).

\section{Gene expression analysis}

For RNASeq analysis, RNA was prepared from hypocotyls or the upper $1 \mathrm{~cm}$ of control and infected plants 16 and 26 DPI. Three independent biological replicates were used for the transcriptomic analysis and a second set of three biological replicates, grown at a different time, used for qRTPCR analysis. RNASeq was 
performed at the Applied Genomics and Analytical Technologies Dept., National Research Council of Canada using an Illumina Hi-Seq 2500 platform. Approximately 25 million reads were obtained from each sample. Paired end reads were mapped using Stampy (Lunter and Goodson 2011) to the Arabidopsis TAIR10 genome (https://www.arabidopsis.org/) and $P$. brassicae genome (Rolfe et al. - unpublished). Over $99 \%$ of the reads matched one genome or the other. For the 16 DPI samples $\sim 13 \%$ (3.5 million) of the reads matched the $P$. brassicae genome in both hypocotyl and root samples. For the 26 DPI samples, $48 \%$ of the reads (12 million) matched the $P$. brassicae genome from hypocotyl tissue and $34 \%$ (9 million) reads from root tissue. Reads matching predicted transcripts were counted using HTSeqCount (Anders et al. 2015) using the default 'union' method and differential gene expression analysed using DESeq2 (Love et al. 2014).

Phylogenetic trees were constructed using MEGA6 (Tamura et al. 2013). Fold-change data were overlaid on metabolic pathways using Mapman (Usadel et al. 2009).

Quantitative RT-PCR was performed as described in Malinowski et al. (2012) using the following primers ARR4 (At1g10470) forward 5'-GCCTGGAATGAC TGGTTATGAG-3', reverse 5'-AGAAATCTTGAGCA CCTTCCTC-3'; ARR5, (At3g48100), forward 5'-CTT CATTAGCATCACCGAAACTTC-3', reverse 5'-AAG CTCTTGTCGCACTATCG-3'; ARR6, (At5g62920), forward 5'-GTCGGTTTTGAGGATTTGAAGG-3', reverse 5'-AGGCAAAATGTTCTCGGAGG-3', ARR7, (At1g19050), forward 5'-CGTAAAGTCATCGAGAGG TTGC-3', reverse 5'-TCCGTCACTATCAAATTCA CCTTC-3'. All results were normalised against $18 \mathrm{~S}$ rRNA expression.

A microarray analysis of control and infected ipt1;3;5;7 tissue was performed using control and infected root and hypocotyl tissue 16 DPI. RNA was prepared from duplicate samples and hybridised to Affymetrix Aragene-1-1-ST microarrays using the NASC International Affymetrix Service (http:// affymetrix.arabidopsis.info/). Expression data contained in the CEL files provided from this service were normalised using the $\mathrm{R}$ package 'oligo' with the RMA option (Carvalho and Irizarry 2010) and differential expression calculated using 'limma' (Smyth 2005). Data are available from ArrayExpress (www.ebi.ac.uk/arrayexpress) under the accessions E-MTAB-4201 (microarray) and E-MTAB-4176 (RNA-Seq).
Quantification of auxins and cytokinins

For analysis of cytokinins $\sim 50 \mathrm{mg}$ of fresh material was collected (hypocotyl $+1 \mathrm{~cm}$ root) and frozen immediately in liquid nitrogen. Samples were extracted in modified Bielesky buffer (methanol/water/formic acid, 15/4/ $1, v / v / v ;$ Hoyerová et al. 2006) using an internal standard of stable isotopically labelled cytokinins $(0.5 \mathrm{pmol}$ of $\mathrm{CK}$ bases, ribosides, $\mathrm{N}$-glucosides, 1 pmol of $O$-glucosides and nucleotides). Material was extracted by bead beating in a Precellys tube (Bertin Technologies) containing $2 \times 3 \mathrm{~mm}$ diameter tungsten carbide beads. Samples were beaten for $2 \times 5 \mathrm{~min}$ at $27 \mathrm{~Hz}$ with cooling in between. All other extraction procedures followed Svačinová et al. (2012), including modifications described by Antoniadia et al. (2015). Auxin measurements were made from $\sim 20 \mathrm{mg}$ of material extracted in $50 \mathrm{mM}$ Na-phosphate buffer $\mathrm{pH} 7$ including $1 \%$ diethyldithiocarbamic acid with the same bead beating procedure as above according to Novák et al. (2012). All samples were analysed by ultra-high performance liquid chromatography (UHPLC) (Acquity UPLC ${ }^{\circledR}$; Waters, Milford, MA, USA) coupled to a Xevo ${ }^{\circledR}$ TQ-S (Waters, Milford, MA, USA) triple quadrupole mass spectrometer equipped with an electrospray interface.

\section{Results}

Cytokinin biosynthesis, response and degradation

To better understand the transcriptional changes associated with the initiation of gall formation (16 DPI) and later stages of disease development (26 DPI) we undertook an RNASeq analysis of RNA isolated from control and infected tissue. Due to the spatial and temporal complexity of the infection (Malinowski et al. 2012), tissue was collected from hypocotyls separately from the upper $1 \mathrm{~cm}$ of the root system. Independent measurements of the expression of selected genes by qRTPCR produced results comparable to the RNASeq experiment (Supplementary Table 1). Table 1 shows the expression of genes associated with host cytokinin biosynthesis, response and degradation. Results have been ranked by expression and are presented as LOG2 foldchange relative to uninfected control tissue.

The expression of numerous genes involved in cytokinin biosynthesis was strongly down-regulated in both the hypocotyls and upper parts of the root system and at 
Table 1 Expression of genes associated with cytokinin biosynthesis, response and degradation in clubroot infected tissue. Results are from RNASeq experiments and are presented as the $\log _{2}$ fold-change between control and infected hypocotyl $(\mathrm{H})$ and root
(R) tissue ( $p<=0.05$, corrected for false discovery) at 16 and 26 DPI. Expression is the average fpkm in all samples. Each sample contained 3 biological replicates

\begin{tabular}{|c|c|c|c|c|c|c|}
\hline AGI & Gene & $16 \mathrm{H}$ & $16 \mathrm{R}$ & $26 \mathrm{H}$ & $26 \mathrm{R}$ & Expression \\
\hline \multicolumn{7}{|l|}{ Synthesis } \\
\hline Atlg67110 & CYP735A2 & ns & 0.60 & -1.75 & $\mathrm{~ns}$ & 31.17 \\
\hline At5g38450 & CYP735A1 & -4.33 & -1.33 & -4.53 & -4.61 & 6.83 \\
\hline At3g63110 & IPT3 & -3.11 & -1.72 & -3.69 & -2.26 & 73.43 \\
\hline At5g19040 & IPT5 & -2.68 & -1.74 & -3.16 & -3.38 & 13.93 \\
\hline Atlg68460 & IPT1 & 1.79 & 4.01 & ns & 3.58 & 3.37 \\
\hline At3g23630 & IPT7 & 1.73 & ns & ns & 1.98 & 3.13 \\
\hline \multicolumn{7}{|c|}{ IPT4,6,8 not expressed } \\
\hline At5g20040 & IPT9 & ns & ns & ns & ns & 8.59 \\
\hline At2g27760 & IPT2 & ns & ns & ns & 1.68 & 5.93 \\
\hline At5g11950 & LOG8 & ns & ns & ns & -0.52 & 20.28 \\
\hline At2g28305 & LOG1 & -2.33 & -1.70 & ns & -3.61 & 3.87 \\
\hline At2g37210 & LOG3 & ns & ns & ns & ns & 3.36 \\
\hline At5g06300 & LOG7 & ns & ns & ns & ns & 2.70 \\
\hline At4g35190 & LOG5 & -4.05 & -2.05 & -3.86 & -2.25 & 1.60 \\
\hline At5g03270 & LOG6 & -6.14 & -4.36 & -4.47 & -4.55 & 1.51 \\
\hline At3g53450 & LOG4 & ns & ns & -1.95 & -1.38 & 0.98 \\
\hline \multicolumn{7}{|c|}{ LOG2,9 not expressed } \\
\hline \multicolumn{7}{|l|}{ Response } \\
\hline \multicolumn{7}{|l|}{ A type ARRs } \\
\hline At1g10470 & ARR4 & -1.68 & -1.24 & -1.74 & -1.94 & 10.21 \\
\hline At3g48100 & ARR5 & -3.01 & -1.91 & -4.46 & -2.76 & 8.81 \\
\hline At3g57040 & ARR9 & -2.46 & -1.53 & -1.99 & -0.94 & 7.53 \\
\hline At1g19050 & ARR7 & -1.71 & -1.56 & -1.29 & -0.73 & 7.46 \\
\hline At5g62920 & ARR6 & -1.98 & -1.73 & -1.89 & -1.21 & 7.45 \\
\hline At2g41310 & ARR8 & -1.45 & ns & -1.76 & $\mathrm{~ns}$ & 7.24 \\
\hline At1g74890 & ARR15 & -1.99 & -3.23 & -1.82 & -2.15 & 5.97 \\
\hline At1g59940 & ARR3 & 4.06 & 1.84 & 2.34 & 2.84 & 2.89 \\
\hline At2g40670 & ARR16 & ns & 1.48 & -2.10 & 2.63 & 2.55 \\
\hline \multicolumn{7}{|c|}{ ARR17,22 not expressed } \\
\hline \multicolumn{7}{|l|}{ Degradation } \\
\hline At5g21482 & CKX7 & ns & ns & ns & -0.67 & 8.10 \\
\hline At3g63440 & CKX6 & -1.32 & -1.43 & -1.49 & -1.56 & 7.37 \\
\hline Atlg75450 & CKX5 & ns & 0.43 & ns & 0.56 & 6.77 \\
\hline At2g41510 & CKX1 & -2.46 & -4.04 & -2.00 & -2.55 & 5.27 \\
\hline At4g29740 & CKX4 & -1.50 & -1.76 & ns & ns & 3.38 \\
\hline At5g56970 & $\mathrm{CKX} 3$ & -3.92 & -1.95 & ns & -1.92 & 1.86 \\
\hline CKX2 not exp & & & & & & \\
\hline
\end{tabular}

Not expressed (less than 1 count per fpkm)

$n s$ no significant difference in expression 
both 16 and 26 DPI. In particular, the expression of the adenine isopentenyltransferase genes IPT3 and 5 was greatly reduced and these represented the most strongly expressed adenine IPTs in these tissues. Figure 1 shows the expression of IPT5::GUS constructs in control and infected hypocotyl tissue. In control plants IPT5::GUS expression was evident in phloem bundles developing exterior to the vascular cambium (VC) but expression was much reduced in infected plants and restricted to phloem bundles associated with small islands of xylem. Whilst small increases in IPT 1 and 7 expression were observed, the overall expression of these genes was much lower and would have contributed relatively little to CK synthesis. Reduced expression was also observed for CYP735A1 and 2 which convert isopentenyladenine nucleotides to the corresponding trans-zeatin molecules and $L O G$ genes associated with the production of active CK species from CK ribosides.

Cytokinin acts via a series of receptors $(A H K 1-4)$ all of which showed little change in expression upon infection (fold-changes were not significant in either tissue or time-point except 26 DPI in root tissue where there were very small increases ( $\sim 1.5$-fold change) in the expression of $A H K 1-3)$. Likewise, there was little or no change in the expression of downstream AHP signalling components (data not shown). Arabidopsis contains two classes of downstream response regulators, type A and B ARRs. Type A ARRs are responsive to cytokinin and the majority were strongly repressed upon infection. Of those which were significantly expressed in the root or hypocotyl (ARR 4-9) all were repressed upon infection. $A R R 3,15$, and 16 did show increased expression in some samples but were only weakly expressed in these tissues.

As the majority of A type ARRs were strongly downregulated in these experiments (and in an independent experiment - see also Fig. 5) but had previously been reported as up-regulated upon clubroot infection (Siemens et al. 2006), we used ARR5::GFP reporter:fusion constructs to visualise cytokinin responsive gene expression in clubroot infected tissue. Handcut cross-sections were made through control and infected hypocotyl tissue and GFP expression visualised by epifluorescence microscopy. In uninfected Arabidopsis plants, ARR5::GFP was expressed in growing primary root tips (Fig. 2a) and also in tissue undergoing secondary thickening (Fig. 2b, c). Cross-sections through hypocotyls of control plants showed expression in phloem bundles exterior to the $\mathrm{VC}$ forming a near continuous ring (Fig. 2d-f - upper panel). In infected tissue, expression was still evident and associated with phloem bundles developing from the VC (Fig. 2d-f, lower panels). However, expression became punctate as tissue morphology was progressively distorted by the presence of hypertrophic cells infected with plasmodia and at later stages of development, spores. Given the increase in the total cross-sectional area of the infected tissue, the extent of ARR5::GFP expression in infected tissue was less than that seen in controls, consistent with the transcriptomic data. There was no
Fig. 1 Expression of IPT5::GUS in Arabidopsis at 32 DPI. Cross sections through the hypocotyls of control plants (a, c) showed GUS expression in the vicinity of phloem. In infected (b, d) plants expression is restricted to a few phloem parenchymal cells (arrow) in regions where isolated islands of meristematic activity and vascular development occur. $P h$ phloem, $X y$ xylem, $P b P$. brassicae spores. Scale bars $=$ $50 \mu \mathrm{m}$

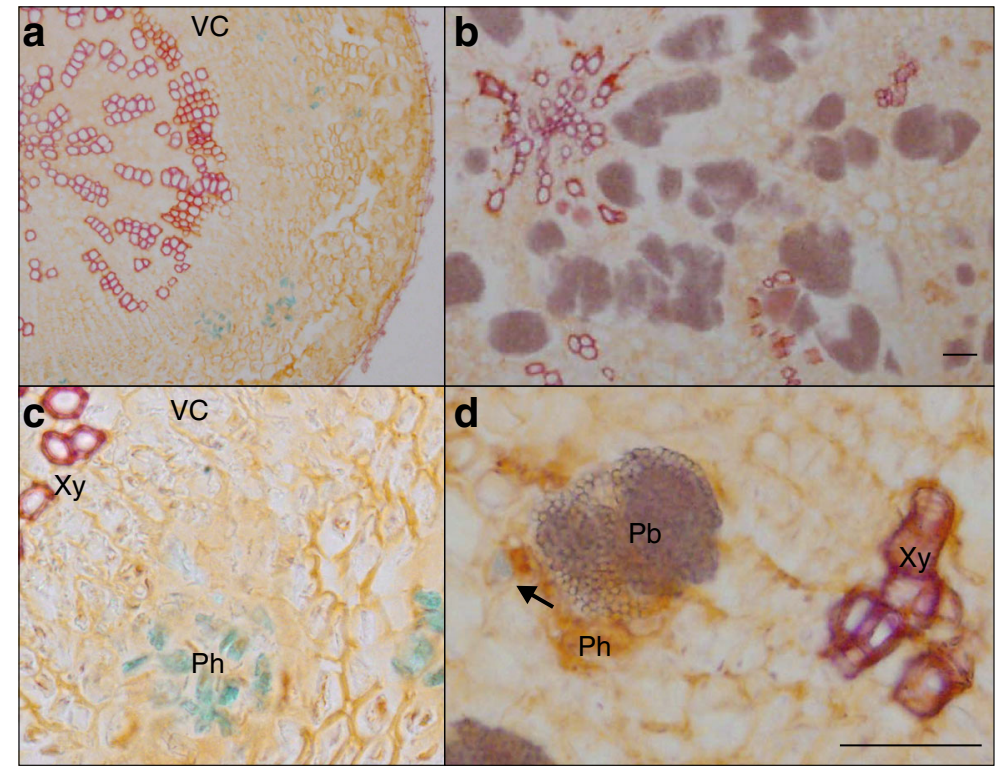



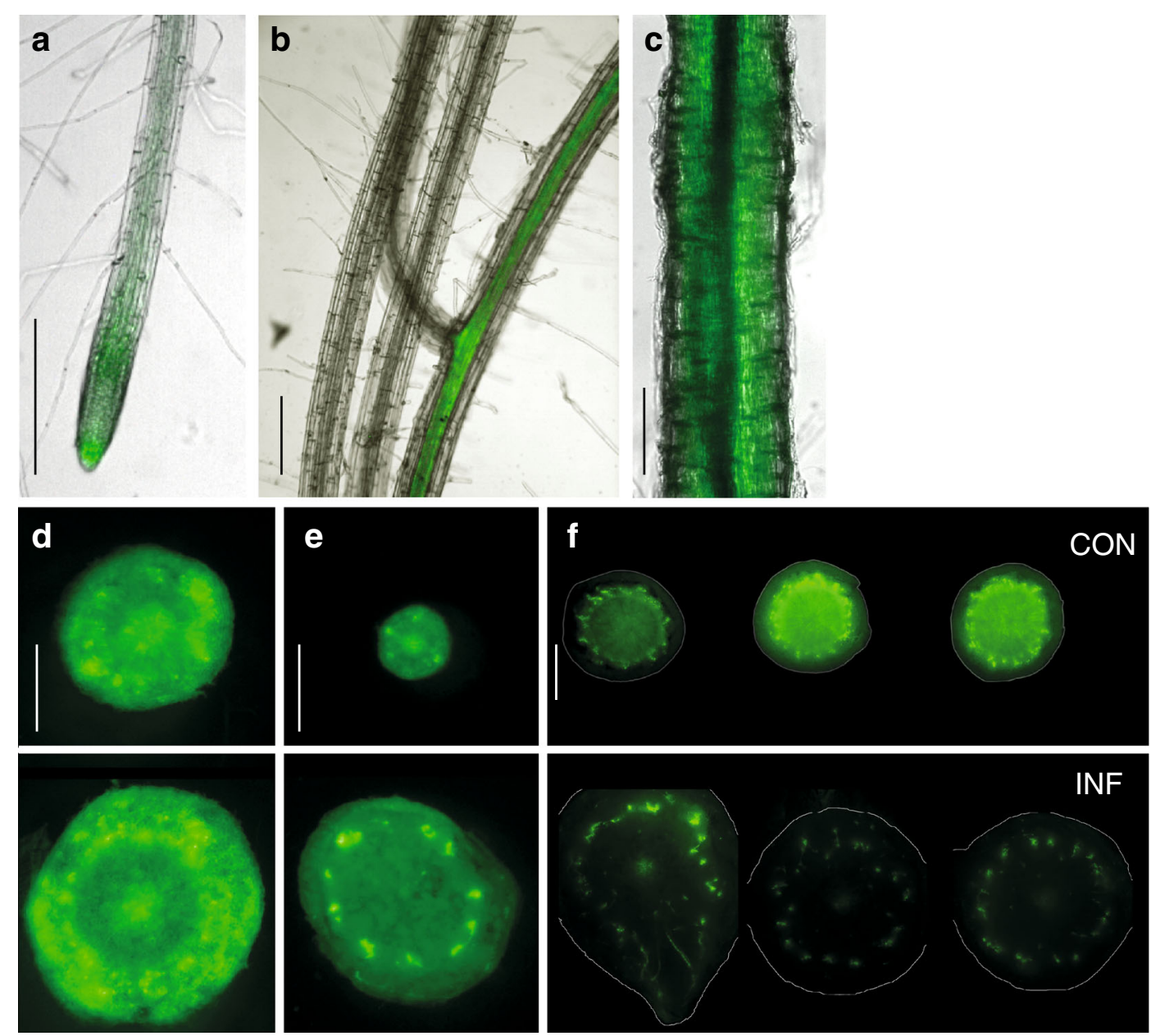

Fig. 2 Expression of ARR5::GFP in Arabidopsis. a-c uninfected 18 day-old plants showing ARR5::GFP expression in root tips, roots undergoing secondary thickening and hypocotyls. $\mathbf{d}-\mathbf{f}$ are cross-sections through hypocotyls of uninfected (upper) and

infected (lower) plants 11, 15 and 28 DPI. The extent of the hypocotyl in infected plants has been highlighted for clarity. Scale bars: a-d $100 \mu \mathrm{m}, \mathbf{e}-\mathbf{f} 1 \mathrm{~mm}$. All images were taken under the same illumination conditions and identical exposure times

evidence of ARR5::GFP expression in regions outside of the developing phloem indicating that the overall pattern of expression had not altered.

The P. brassicae genome encodes two IPT genes

The recent completion of the $P$. brassicae genome ((Schwelm et al. 2015) and Rolfe et al. - unpublished) has allowed the identification of two P. brassicae genes predicted to encode isopentenyltransferases. These have been named PbIPT1 (PbPT3Sc00040_Am_6.202_1) and PbIPT2 (PbPT3Sc00024_Sm_2.181_1). PbIPT1 is identical to the hypothetical protein PBRA_004561 reported by Schwelm et al. 2015) but PbIT2 is only $94 \%$ similar to PBRA_005817. Supplementary Fig. 1 shows a

dendogram of their relatedness to other known plant and bacterial IPT genes. Given the relatively low homology to known sequences it is not possible to determine whether these genes use tRNA or adenine nucleotides as substrates (i.e. synthesise $c Z$ or $t Z$ ). Both genes were expressed in roots and hypocotyls (Supplementary Fig. 2) and there was no evidence for differential expression between the time points tested. The P. brassicae genome also contained a complete pathway for the synthesis of isopentenylpyrophosphate from acetyl coA via the mevalonate pathway and conversion to dimethylallylpyrophosphate by isopentenyldiphosphatedelta-isomerase. Together these data indicate that P. brassicae has the capacity to synthesise CKs and that these genes are expressed during gall formation. 
The cytokinin content of control and clubroot-infected galls

The complex nature of cytokinin biosynthesis, conjugation and degradation and the instability of many of these compounds have made analytical studies of their concentrations in small amounts of tissue challenging. However, developments in analytical procedures and the use of labelled isotopes of individual components now allows the spectrum of CK related molecules to be quantified in milligram amounts of tissue. We therefore measured the CK metabolite content of control and infected roots + hypocotyls as the same timepoints as the transcriptomic analysis allowing direct comparisons to be drawn.

Supplementary Table 2 contains all of the measurements made with selected data shown in Fig. 3.

As expected, trans-zeatin ( $t Z$ ) and isopentenyladenine (iP) were the major active CKs found in both uninfected and infected tissue. cis zeatin (cZ) and dihydrozeatin (DHZ) concentrations were $\sim 100$ fold lower (Fig. 3). The concentrations of both $t \mathrm{Z}$ and iP were significantly lower in infected tissue at both timepoints, as were the concentrations of $c \mathrm{Z}$ and DHZ. Similar reductions were seen in the concentrations of the precursor ribosides (at both 16 and $26 \mathrm{DPI}$ ) and the nucleotides (but only at $26 \mathrm{DPI}$ ). The concentrations of conjugated $\mathrm{N}$-glucosides were reduced to a similar extent. Figure 4 shows metabolic pathways integrating the transcriptomic and metabolic data for hypocotyls 16 and 26 DPI (the root tissue showed similar patterns). The overall pattern was very clearly one of repression - the majority of host CK metabolic genes and metabolites showed significant reductions in expression and concentration in infected tissue. It was noticeable that although the majority of degradative genes were repressed, there was a small increase in the expression of the O-glucoside transferases ( $t Z \mathrm{ZOGT}$ and cZOGT) at both time points with corresponding small increases in the O-glucoside conjugates, $t \mathrm{ZOG}$ and cZROG, at 26 DPI. In contrast to cytokinin, the auxin content of tissue was not affected by infection (Supplementary Fig. 3).

Infection of plants with altered CK biosynthesis influences disease development

The availability of extensive genetic resources in Arabidopsis allowed us to examine the impact of reducing host CK biosynthesis on disease development. The quadruple isopentenyltransferase mutant ipt1 ipt3 ipt5 ipt7 has severely reduced cytokinin content (Miyawaki et al. 2006), does not form cambium and undergoes virtually no secondary thickening of the root or hypocotyl (Matsumoto-Kitano et al. 2008). Fourteen day old ipt1;3;5;7 plants were infected with $P$. brassicae and anatomical changes were scored 26 and 36 DPI. Figure 5 shows control and infected ipt quadruple mutants 26 DPI. Although some hypocotyl swelling in infected plants

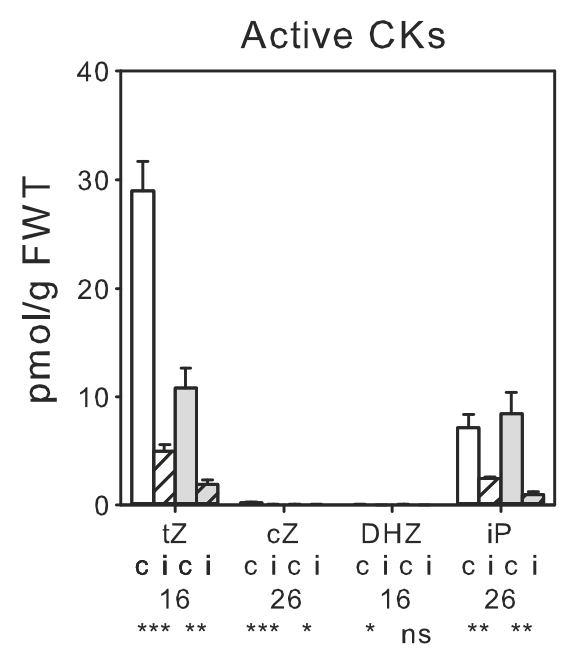

Fig. 3 The cytokinin content of control and clubroot-infected Arabidopsis plants. Active, precursor and conjugated cytokinin content of control (c) and infected (i) root + hypocotyl tissue 16
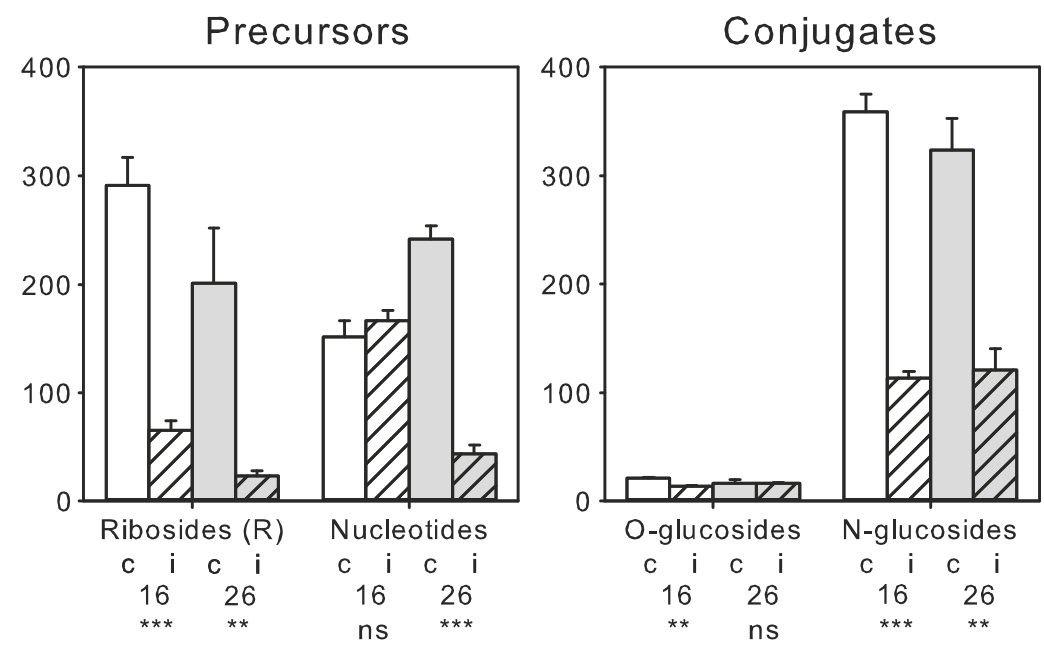

and 26 DPI. Results are the means + SE of 3 independent replicates expressed as pmol g ${ }^{-1}$ fresh weight. Significance is indicated by $* p<0.05, * * p<0.01$ and $* * * p<0.001$ or ns (not significant) 


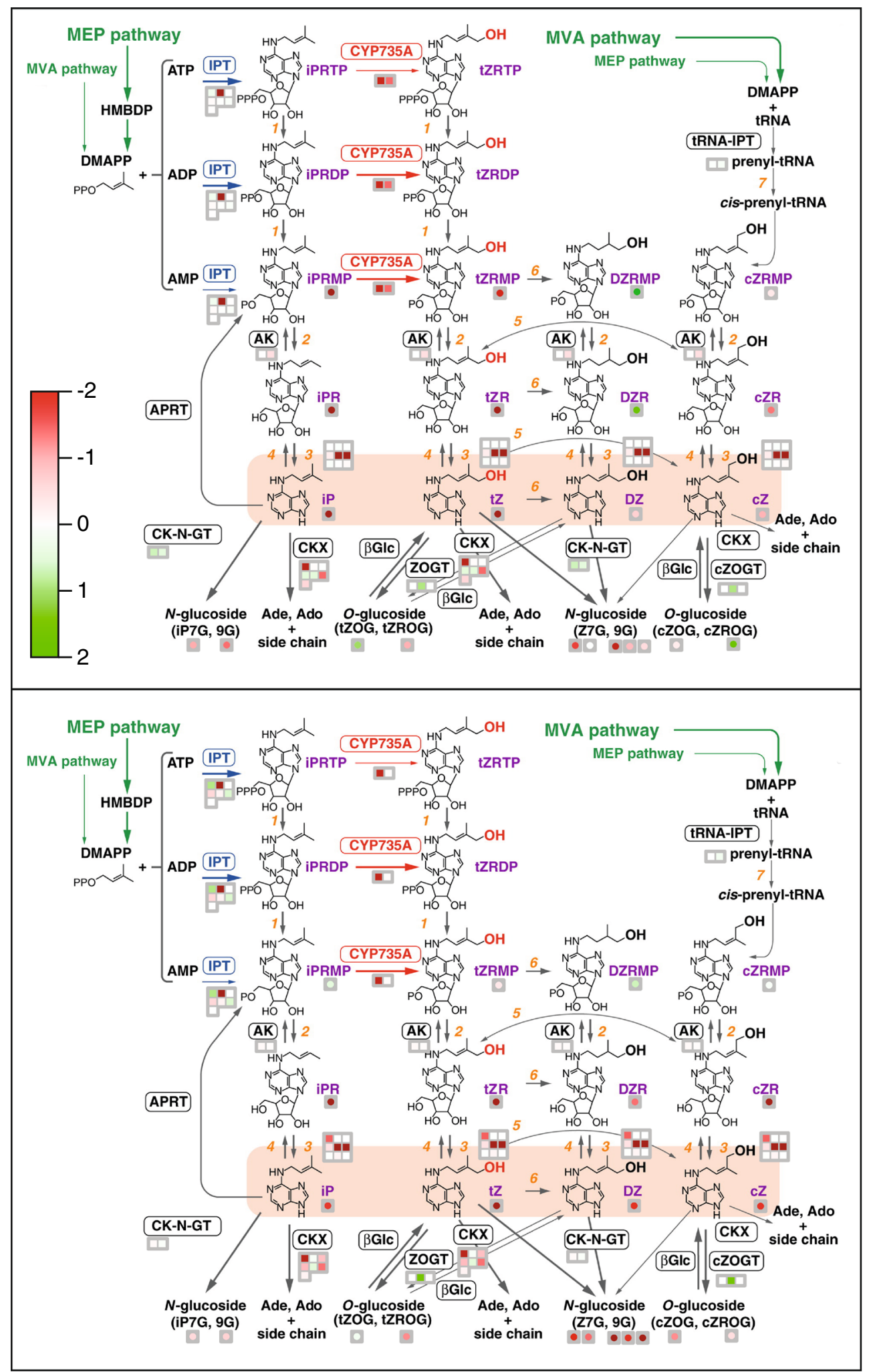


Fig. 4 Metabolic pathways of cytokinin synthesis, metabolism and degradation. Results are LOG2-fold change between control and infected hypocotyl tissue, 16 DPI (upper panel) and 26 DPI (lower panel). The values were overlaid on the metabolic pathway of Sakakibara (2006) using Mapman

was evident, it was much less than that of infected wild type plants - the degree of thickening was similar to that seen in the lateral roots of infected wild type plants. Transverse sections through the hypocotyl tissue of uninfected ipt 1;3;5;7 plants showed that no secondary thickening occurred at any time point (Fig. 5c). Hypocotyl tissue of infected ipt 1;3;5;7 plants showed extensive cell expansion, but very limited cell proliferation and no characteristic islands of fragmented cambium were observed (Fig. 5d). The lifecycle of the pathogen was slowed in the ipt 1;3;5;7 mutant. In infected Col-0 plants, resting spores were present 26 DPI (Malinowski et al. 2012). However, in infected ipt 1;3;5;7 plants, secondary plasmodia were present 26 DPI (Fig. 5e) and resting spore formation was delayed until 36 DPI (Fig. 5f) indicating that the lack of host produced cytokinin and associated loss of secondary thickening led to slower disease development. Pathogen vigour was assessed by collecting spores from infected ipt 1;3;5;7 plants 36 DPI that were then used to inoculate Col-0 plants. The number of spores produced was much lower than that of wild type due to the small size of the mutant plants, but this was corrected for by using the equal numbers of spores for subsequent inoculations. Whilst a significant reduction in disease index (DI) was observed (Table 2), infection still occurred, indicating that a proportion of the $P$. brassicae-infected cells were able to develop viable resting spores despite the lack of secondary thickening in the ipt 1;3;5;7 host plant.

Because P. brassicae has been proposed to have the capacity to synthesise cytokinins (Müller and Hilgenberg 1986), we examined the expression of the cytokinin-responsive genes ARR4-7 in the hypocotyls of Col- 0 and mutant plants 26 DPI. At this time point, $A R R$ gene expression was similar or slightly lower in the hypocotyls of infected wild type plants compared to uninfected controls. Expression of these cytokininresponsive genes was low in uninfected ipt 1;3;5;7 quadruple mutants, but was restored to wild type levels (or above) upon infection (Fig. $5 \mathrm{~g}$ ). Thus, infection with
Plasmodiophora leads to a cytokinin-signalling response, but this induction was insufficient to rescue the phenotype of the ipt 1;3;5;7 mutant. A microarray study of control and infected ipt 1;3;5;7 mutants showed that there was no increase in expression of any of the host IPT genes upon infection, hence $A R R$ expression did not result from a compensatory increase in expression of other IPT genes (Supplementary Table 3).

\section{Discussion}

This report brings together advanced analytical methods for CK quantification with a detailed analysis of both the host and pathogen transcriptome providing new insights into hormonal changes associated with clubrootinduced gall formation. Malinowski et al. (2012) found that $P$. brassicae hijacks the development of the secondary thickening process in infected Arabidopsis plants causing an activation of $\mathrm{VC}$ activity, a strong repression of xylogenesis but a maintenance of phloem production. Interpretation of the results must consider therefore whether they occur as a consequence of these developmental changes or are driving them. For example, although phloem production is maintained during gall formation, the expression of phloem-specific genes such as CLE41/44 appears slightly repressed ( 2 -fold) as a consequence of an increased proportion of undifferentiated cells (Supplementary Table 1). In contrast, xylogenesis is much more strongly repressed and genes associated with the regulation of these processes show an $\sim 8$-fold reduction at 16 DPI.

The regulation of host CK metabolism and response in developing galls

We have demonstrated that there was a strong repression of host gene expression associated with CK biosynthesis, signalling and degradation at 16 DPI and 26 DPI in both infected root and hypocotyl tissue. Isopentenyltransferases (IPTs) are the rate limiting steps in CK biosynthesis (El-Showk et al. 2013) and Arabidopsis contains two classes of IPTs: AtIPT1, 3, 4-8 encode forms that use ADP/ATP as substrates whilst AtIPT2 and 9 encode forms that use tRNA as substrates (Miyawaki et al. 2006). The ADP/ATP IPTs synthesise the active cytokinins iP and $t \mathrm{Z}$ whilst the tRNA IPTs synthesise $c Z$ which shows limited activity in Arabidopsis (Gajdošová et al. 2011). IPT3 and IPT5 

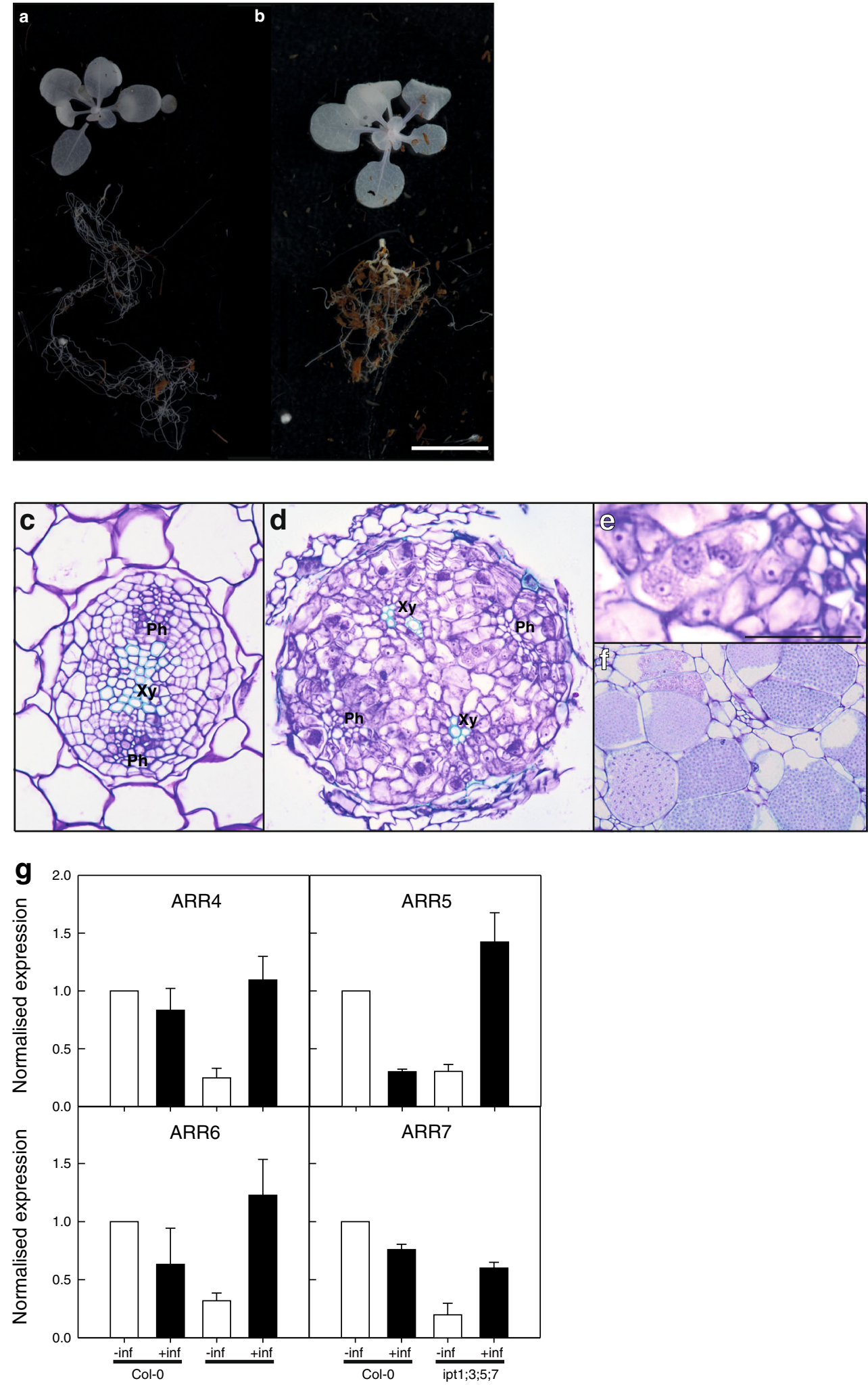
4 Fig. 5 Infection of ipt1,3,5,7 quadruple mutant. Control (a) and infected (b) plants 26 DPI. Some limited swelling is evident in the hypocotyls of infected plants. Cross-sections through hypocotyls of control (c) and infected (d) plants 26 DPI. Xy xylem, Ph phloem. Plasmodial development in ipt 1;3;5;7 mutants 26 DPI (e) and 36 DPI (f). Expression of A-type ARR genes in control and infected plants (g) assessed by qRTPCR. Results are the means of 3 replicates + SE. Expression was normalised uninfected Col-0 plants. Scale bars: a, b $1 \mathrm{~cm}, \mathbf{c}-\mathbf{f} 50 \mu \mathrm{m}$

were the most abundantly expressed IPT genes in root and hypocotyl tissue and their expression was strongly repressed in infected tissue $(\sim 8$-fold $)$ at both time points. This reduction was likely to result from transcriptional regulation rather than alterations in the proportion of specific cell types. Although the expression of IPTI and IPT7 was induced, the transcript abundance was much lower than that of IPT3 and IPT5 and was unlikely to contribute significantly to CK synthesis in this tissue. The expression of IPT2 and IPT9 was also low in these tissues and showed little change during gall formation. The $c Z$ content of the tissue was extremely low supporting the view that $c Z$ plays little role in rapidly growing tissue in Arabidopsis. The expression of many other genes associated with host CK biosynthesis (CYP735A1/2 and LONELY GUY $L O G)$ was also repressed. Interestingly, whilst the expression of many cytokinin oxidases was repressed, genes associated with CK conjugation showed either no alteration in expression or tended to be up-regulated slightly, indicating that their regulatory pathways were distinct from that of other elements of CK metabolism.

The repression of genes associated with CK metabolism was reflected in the expression of genes associated with CK response (type A $A R R$ genes). Cytokinins are perceived by a two-component signalling circuit with the type A ARR genes forming a negative feedback loop

Table 2 Infectivity of clubroot spores isolated from wildtype and ipt mutant plants. Plants (Col-0) were inoculated with $2 \times 10^{6}$ spores collected from infected plants and scored for symptoms 32 DPI. The scoring scale and calculation of disease index is described in Siemens et al. (2002)

\begin{tabular}{lll}
\hline Origin of spores & Symptom score $+/-$ SD & Disease index (\%) \\
\hline Col-0 & $3.33+/-0.82$ & 83 \\
ipt $1 ; 3 ; 5 ; 7$ & $1.73+/-1.39$ & 43 \\
\hline
\end{tabular}

(Hwang and Sheen 2001) - expression of these genes is closely correlated with CK content and ARR5 is used routinely as a CK-responsive promoter:reporter fusion (Romanov et al. 2002). In our study we found type A $A R R$ gene expression to be strongly repressed. This was also evident in cross-sections of ARR $5:: G F P$ plants in which expression was closely associated with developing phloem and expression fell as gall formation was initiated 16 DPI.

Our results are in broad agreement with previous studies in Arabidopsis and other Brassicas where CK metabolism has been reported to be repressed at the onset of gall formation. For example, Ando et al. (2005) found that the expression of IPT genes in Chinese cabbage fell 20 DPI in regions of the root system where gall formation was initiated (but was upregulated in areas where galls had yet to form). Likewise Agarwal et al. (2011) found that the expression of CK biosynthetic genes was down-regulated 4, 7 and 10 DPI in Arabidopsis - they also found that although the expression of CK glycosylation genes was downregulated at 4 and 7 DPI, expression increased at 10 DPI consistent with our observations at 16 DPI. Siemens et al. (2006) performed a microarray analysis of gene expression in control and infected Arabidopsis root and hypocotyl tissue at 10 and 23 DPI. In most respects their results are similar to our findings - CK biosynthesis and degradation were down-regulated during gall formation. However, they reported an increase in ARR5 and ARR 10 expression and an up-regulation of the CK receptor $C R E 1 / A H K 4$ at $10 \mathrm{DPI}$. This difference might result from the slightly earlier time point studied at 10 DPI there was little cell expansion in the infected tissue sampled but the response was also very small - a 2.3-fold increase in transcript abundance without corresponding changes in expression of other A-type $A R R$ genes. Jubault et al. (2013) also performed a microarray analysis of infected Arabidopsis tissue and found that AtIPT9 and ARR4 gene expression was repressed at 7 DPI. A recent study by Schuller et al. (2014) shows that there are highly localised changes in gene expression that may not be reflected in transcriptional measurements of RNA isolated from bulk tissue. This study used laser microdissection of clubroot infected Arabidopsis root tissue to isolate individual cells containing small or large plasmodia at 14 and 21 DPI followed by transcriptomic analyses. Whilst the repression of $C K X 1, C K X 3$ and $C K X 7$ was confirmed, $C K X 6$ showed a more complicated pattern. $C K X 6$ expression 
was down-regulated in the infected central cylinder but was strongly up-regulated in cortical cells containing plasmodia. Clearly a combination of detailed microscopy of reporter:promoter fusions and single-cell analysis techniques such as described in Schuller et al. (2014) will be important in unravelling the mechanistic basis of gall formation.

The development of advanced microanalytical techniques has allowed us to obtain a precise quantification of CKs, their precursors and derivatives in milligram quantities of control and infected Arabidopsis tissue. The reproducibility of the system was excellent with relative standard deviations typically $<10 \%$ from 3 replicates. This represents the first complete overview of CK metabolism in P. brassicae-infected Arabidopsis tissues. The concentration of active $\mathrm{CKs}$, CK ribosides and $\mathrm{N}$-glucosides was significantly reduced at both 16 and 26 DPI. However, the reduction in $t Z$ was much greater than $i P$ at 16 DPI. The $t Z$ content of infected tissue 16 DPI was $17 \%$ of that of controls whilst the $i P$ content was $34 \%$. This is consistent with acropetal $t Z$ transport via the xylem and basipetal $i P$ transport via the phloem (Bishopp et al. 2011). The impact on $t Z$ is likely to have been greater as xylogenesis is strongly repressed, as is root development during gall formation.

Several early studies found that the CK content of clubbed Brassicas increased. For example, Müller and Hilgenberg (1986) found that extracts of clubbed tissue from Chinese cabbage contained large amounts of $c Z$ and $c Z$ riboside unlike Arabidopsis where the cis forms are minor components. Devos et al. (2005) also studied Chinese cabbage and found the zeatin content to be increased at early time points, falling later although they did not distinguish between the cis and trans forms. Devos et al. (2006) also made measurements of the CK content of infected Arabidopsis tissue at 4 DPI. They found a small (but not statistically significant) increase in zeatin and a small increase in $\mathrm{iP}$ at this time point potentially indicating a role for $\mathrm{CK}$ at the very earliest stages of $P$. brassicae infection but well before the development of the VC and onset of gall formation. Clearly species differences and the time points studied greatly influence the responses observed.

Against this background of a decrease in active CKs in Arabidopsis, some precursors and conjugates showed less marked responses. The CK nucleotide content of infected tissue did not differ significantly from that of controls at 16 DPI. The dominant CK nucleotides were iPRMP which showed a significant increase at this time point whilst $t$ ZRMP showed a small decrease. These results suggest that at the onset of gall formation synthesis of iP nucleotides continued, but that conversion to the $t Z$ forms by CYP735A1/A2 or to the ribosides by adenosine kinase, was restricted to some extent. The impact on the N-glucoside conjugates was also much greater than the O-glucoside conjugates. Whilst Oglycosylation is reversible and CK-O-glucosides are storage products (Mok et al. 1992), N-glycosylation is irreversible and N-glucosides are degradation products (Letham et al. 1983; Vankova 1999). These results are consistent with a decrease in all aspects of CK biosynthesis but also the degradation pathways whether via $\mathrm{CK}$ oxidases or $\mathrm{N}$-glycosylases.

Our results show clearly that CK synthesis and response are down-regulated from 16 DPI onwards, the point at which gall formation is initiated in Arabidopsis. Therefore we propose that this decrease in CK content may be an important contributor to the regulation of host development during gall formation. During primary root formation $\mathrm{CK}$ content, as visualised by $A R R 5$ promoter::reporter fusions, is initially high but then falls as the primary roots mature. The CK response pathway is activated again as the VC develops and secondary thickening commences with ARR5 expression greatest in the vicinity of developing phloem bundles. Only once the $\mathrm{VC}$ is present can gall formation commence and is associated with a reduction in CK content. This model is consistent with the findings of Siemens et al. (2006) that gall formation is reduced in plants constitutively expressing CK oxidase as in these plants the reduction in CK content will slow the formation of the VC and this slow gall formation.

What contribution does $P$. brassicae make to the CK content of infected tissue?

The P. brassicae genome contains 2 IPT genes. Although these genes show greatest sequence homology to tRNA IPTs, their substrate specificity remains to be characterised. RNASeq analysis demonstrated that these genes were expressed at both 16 and 26 DPI hence they may have contributed to the CK content of infected tissue. If these IPTs synthesise $c Z$ then the amounts released must have been very small as the $c Z$ content of uninfected Arabidopsis tissue was low and fell yet further upon infection.

Analysis of infection in the ipt 1;3;5;7 mutant allows us to unravel the relative contributions of the host and 
pathogen to $\mathrm{CK}$ metabolism. CK biosynthesis was extremely compromised in this mutant and, as a consequence, ARR4-7 gene expression was much reduced. Although these plants could develop to a limited extent, the lack of CKs prevented the VC from forming and the roots retained primary root morphology. When these mutants were infected with P. brassicae, ARR4-7 gene expression was induced but the phenotype was not rescued (i.e. no VC developed and no secondary thickening occurred). This was not due to a compensatory increase in expression of other host IPT genes, as a microarray analysis of control and infected ipt 1;3;5;7 tissue showed no change in expression. Müller and Hilgenberg (1986) found that isolated plasmodia were able to take up radiolabelled adenine and make small amounts of a compound that they tentatively assigned as $t Z$. This is consistent with a small amount of $t Z$ being released, stimulating type A $A R R$ genes expression in the ipt 1;3;5;7 mutant but in insufficient quantities to rescue the mutant phenotype by restoring VC development. Also, the pattern of ARR 5::GFP expression in infected wild type plants remained associated with developing phloem bundles hence $\mathrm{CK}$ production could not have been widespread. Therefore we propose that whilst there is evidence for CK production by P. brassicae, the amounts produced have relatively little impact on the development of its host.

Even though disease development and gall formation was restricted in the ipt 1;3;5;7 mutant, infected cells still exhibited significant hypertrophy even though hyperplasia had been abolished. Therefore other phytohormones are implicated in the extensive cell expansion seen in infected tissue. Although GA is an obvious candidate, treatment with GA inhibitors had no effect on gall formation (Päsold and Ludwig-Müller 2013). Recently Schuller et al. (2014) found that brassinosteroid (BR) biosynthesis was up-regulated in enlarged cells and that mutations in the BR receptor (bril-6) and treatment with BR biosynthesis inhibitors reduced gall formation. Given the importance BR and other hormones such as strigolactones in regulating cambial activity and their extensive interactions with auxin and cytokinin (Nieminen et al. 2015) a detailed microscopic analysis of host and plasmodial development in plants with mutations in these signalling pathways is likely to be informative.
Cytokinins are required for $P$. brassicae development

Intriguingly CK may have an important role in the development of the pathogen. Malinowski et al. (2012) showed that although VC activity is required for gall formation it is not required for infection and development of the pathogen. In plants expressing an inducible cell cycle inhibitor ICK1/KRP or bearing a cle41 mutation which restricts VC activity, infection of host cells still occurred and competent spores produced even though gall formation was severely compromised. In contrast, whilst $P$. brassicae could infect the ipt $1 ; 3 ; 5 ; 7$ mutant, plasmodial development was slowed and the infectivity of spores reduced. This suggests that CKs may directly regulate plasmodial development. This is consistent with the presence and expression of IPT genes in the $P$. brassicae genome, the ability of $P$. brassicae to take up adenine and synthesise limited amounts of $t Z$ and the accumulation of host CKs in isolated plasmodia ( $c Z$ in the case of Chinese cabbage; Müller and Hilgenberg (1986)).

\section{Conclusions}

This work extends the model of Devos et al. (2005). $P$. brassicae requires CKs for its development, synthesising a limited amount and but also taking up CKs from its host. In the host, CKs are essential for the development of the VC, a pre-requisite for secondary thickening and normal plant growth but also a route by which $P$. brassicae can hijack host development for gall formation. Gall formation is associated with a decline in CK content that may help drive the host developmental changes seen in infected tissue, but other phytohormones are involved in the hypertrophy characteristic of the disease. This opens potentially new routes for the control of this important disease - either by interfering with the delivery and uptake of CKs by the pathogen or by increasing the CK content of host tissue during the gall formation stage of clubroot disease.

Acknowledgments This work was supported by the Leverhulme Trust (F/00 118/BN) and Leverhulme International Academic Fellowship (IAF-2013-018). O.N., L.S. and M.S. acknowledge the Ministry of Education, Youth and Sports of the Czech Republic (the National Program for Sustainability I, grant No. LO1204), the Internal Grant Agency of Palacký University (IGA_PrF_2015_021)and the Czech Grant Agency (15-22322S). R.M. was supported by the National Science Centre Poland 
SONATA BIS2 grant No. 2012/07/E/NZ3/00510 “Integrated approach for deciphering the mechanism leading to shift in cell proliferation/differentiation balance accompanying clubroot infection". R.M. is the BIO-TALENT ERA-CHAIR team leader/ holder.

We thank Prof Tatsuo Kakimoto (Department of Biology, Graduate School of Science, Osaka University, Toyonaka, Osaka 560-0043, Japan) for the ipt 1;3;5;7 quadruple mutant and IPT5::GUS lines. We thank Ms. Jody Smith and Mrs. Hana Martínková for their expert technical assistance.

Open Access This article is distributed under the terms of the Creative Commons Attribution 4.0 International License (http://creativecommons.org/licenses/by/4.0/), which permits unrestricted use, distribution, and reproduction in any medium, provided you give appropriate credit to the original author(s) and the source, provide a link to the Creative Commons license, and indicate if changes were made.

\section{References}

Agarwal, A., Kaul, V., Faggian, R., Rookes, J. E., LudwigMueller, J., \& Cahill, D. M. (2011). Analysis of global host gene expression during the primary phase of the Arabidopsis thaliana-Plasmodiophora brassicae interaction. Functional Plant Biology, 38(6), 462-478.

Anders, S., Pyl, P. T., \& Huber, W. (2015). HTSeq-a Python framework to work with high-throughput sequencing data. Bioinformatics, 31(2), 166-169.

Ando, S., Asano, T., Tsushima, S., Kamachi, S., Hagio, T., \& Tabei, Y. (2005). Changes in gene expression of putative isopentenyltransferase during clubroot development in Chinese cabbage (Brassica rapa L.). Physiological and Molecular Plant Pathology, 67(2), 59-67.

Antoniadia, I., Plačkovác, L., Simonovika, B., Doležalc, K., Turnbull, C., Ljunga, K., \& O., N. (2015). Cell-type specific cytokinin distribution within the Arabidopsis primary root apex. Plant Cell, doi:10.1105/tpc.15.00176.

Bishopp, A., Lehesranta, S., Vatén, A., Help, H., El-Showk, S., Scheres, B., Helariutta, K., Mähönen, A. P., Sakakibara, H., \& Helariutta, Y. (2011). Phloem-transported cytokinin regulates polar auxin transport and maintains vascular pattern in the root meristem. Current Biology, 21(11), 927-932.

Carvalho, B. S., \& Irizarry, R. A. (2010). A framework for oligonucleotide microarray preprocessing. Bioinformatics, 26(19), 2363-2367.

Devos, S., Vissenberg, K., Verbelen, J.-P., \& Prinsen, E. (2005). Infection of Chinese cabbage by Plasmodiophora brassicae leads to a stimulation of plant growth: impacts on cell wall metabolism and hormone balance. New Phytologist, 166, 241-250.

Devos, S., Laukens, K., Deckers, P., Van Der Straeten, D., Beeckman, T., Inzé, D., Van Onckelen, H., Witters, E., \& Prinsen, E. (2006). A hormone and proteome approach to picturing the initial metabolic events during Plasmodiophora brassicae infection on Arabidopsis. Molecular PlantMicrobe Interactions, 19(12), 1431-1443.
Dixon, G. R. (2009). The occurrence and economic impact of Plasmodiophora brassicae and clubroot disease. Journal of Plant Growth Regulation, 28(3), 194-202.

El-Showk, S., Ruonala, R., \& Helariutta, Y. (2013). Crossing paths: cytokinin signalling and crosstalk. Development, 140(7), 1373-1383.

Gajdošová, S., Spíchal, L., Kamínek, M., Hoyerová, K., Novák, O., Dobrev, P. I., Galuszka, P., Klíma, P., Gaudinová, A., Žižková, E., Hanuš, J., Dančák, M., Trávníček, B., Pešek, B., Krupička, M., Vaňková, R., Strnad, M., \& Motyka, V. (2011). Distribution, biological activities, metabolism, and the conceivable function of cis-zeatin-type cytokinins in plants. Journal of Experimental Botany, 62(8), 2827-2840.

Hoyerová, K., Gaudinová, A., Malbeck, J., Dobrev, P. I., Kocábek, T., Šolcová, B., Trávníčková, A., \& Kamínek, M. (2006). Efficiency of different methods of extraction and purification of cytokinins. Phytochemistry, 67(11), 1151-1159.

Hwang, I., \& Sheen, J. (2001). Two-component circuitry in Arabidopsis cytokinin signal transduction. Nature, 413(6854), 383-389.

Jubault, M., Lariagon, C., Taconnat, L., Renou, J.-P., Gravot, A., Delourme, R., \& Manzanares-Dauleux, M. (2013). Partial resistance to clubroot in Arabidopsis is based on changes in the host primary metabolism and targeted cell division and expansion capacity. Functional \& Integrative Genomics, 13(2), 191-205.

Kageyama, K., \& Asano, T. (2009). Life cycle of Plasmodiophora brassicae. Journal of Plant Growth Regulation, 28(3), 203211.

Letham, D. S., Palni, L. M. S., Tao, G. Q., Gollnow, B. I., \& Bates, C. M. (1983). Regulators of cell division in plant tissues XXIX. The activities of cytokinin glucosides and alanine conjugates in cytokinin bioassays. Journal of Plant Growth Regulation, 2(1-4), 103-115.

Love, M. I., Huber, W., \& Anders, S. (2014). Moderated estimation of fold change and dispersion for RNA-seq data with DESeq2. Genome Biology, 15(12), 550.

Ludwig-Müller, J., Prinsen, E., Rolfe, S., \& Scholes, J. (2009). Metabolism and plant hormone action during clubroot disease. Journal of Plant Growth Regulation, 28(3), 229-244.

Lunter, G., \& Goodson, M. (2011). Stampy: a statistical algorithm for sensitive and fast mapping of Illumina sequence reads. Genome Research, 21(6), 936-939.

Malinowski, R., Smith, J. A., Fleming, A. J., Scholes, J. D., \& Rolfe, S. A. (2012). Gall formation in clubroot-infected Arabidopsis results from an increase in existing meristematic activities of the host but is not essential for the completion of the pathogen life cycle. The Plant Journal, 71(2), 226-238.

Matsumoto-Kitano, M., Kusumoto, T., Tarkowski, P., KinoshitaTsujimura, K., Václavíková, K., Miyawaki, K., \& Kakimoto, T. (2008). Cytokinins are central regulators of cambial activity. Proceedings of the National Academy of Sciences of the United States of America, 105(50), 20027-20031.

Mithen, R., \& Magrath, R. (1992). A contribution to the life history of Plasmodiophora brassicae: secondary plasmodia development in root galls of Arabidopsis thaliana. Mycological Research, 96(10), 877-885.

Miyawaki, K., Tarkowski, P., Matsumoto-Kitano, M., Kato, T., Sato, S., Tarkowska, D., Tabata, S., Sandberg, G., \& Kakimoto, T. (2006). Roles of Arabidopsis ATP/ADP isopentenyltransferases and tRNA isopentenyltransferases 
in cytokinin biosynthesis. Proceedings of the National Academy of Sciences of the United States of America, 103(44), 16598-16603.

Mok, M., Martin, R., Mok, D., \& Shaw, G. (1992). Cytokinin activity, metabolism and function in Phaseolus. In M. Kaminek, D. Mok, \& E. Zazimalova (Eds.), Physiology and biochemistry of cytokinins in plants (pp. 41-46). The Hague: SPB Academic Publishers.

Müller, P., \& Hilgenberg, W. (1986). Isomers of zeatin and zeatin riboside in clubroot tissue: evidence for trans-zeatin biosynthesis by Plasmodiophora brassicae. Physiologia Plantarum, 66(2), 245-250.

Nieminen, K., Blomster, T., Helariutta, Y., \& Mähönen, A. P. (2015). Vascular cambium development. The Arabidopsis Book, e0177.

Novák, O., Hényková, E., Sairanen, I., Kowalczyk, M., Pospíšil, T., \& Ljung, K. (2012). Tissue-specific profiling of the Arabidopsis thaliana auxin metabolome. The Plant Journal, 72(3), 523-536.

Päsold, S., \& Ludwig-Müller, J. (2013). Reduction of clubroot (Plasmodiophora brassicae) formation in Arabidopsis thaliana after treatment with prohexadione-calcium, an inhibitor of oxoglutaric acid-dependent dioxygenases. Plant Pathology, 62(6), 1357-1365.

Romanov, G. A., Kieber, J. J., \& Schmülling, T. (2002). A rapid cytokinin response assay in Arabidopsis indicates a role for phospholipase D in cytokinin signalling. FEBS Letters, 515(1-3), 39-43.

Sakakibara, H. (2006). CYTOKININS: activity, biosynthesis, and translocation. Annual Review of Plant Biology, 57(1), 431449.

Schuller, A., Kehr, J., \& Ludwig-Müller, J. (2014). Laser microdissection coupled to transcriptional profiling of Arabidopsis roots inoculated by Plasmodiophora brassicae indicates a role for brassinosteroids in clubroot formation. Plant and Cell Physiology, 55(2), 392-411.

Schwelm, A., Fogelqvist, J., Knaust, A., Jülke, S., Lilja, T., Bonilla-Rosso, G., Karlsson, M., Shevchenko, A., Dhandapani, V., Choi, S. R., Kim, H. G., Park, J. Y., Lim,
Y. P., Ludwig-Müller, J., \& Dixelius, C. (2015). The Plasmodiophora brassicae genome reveals insights in its life cycle and ancestry of chitin synthases. Scientific Reports, 5 . doi:10.1038/srep11153.

Siemens, J., Nagel, M., Ludwig-Müller, J., \& Sacristán, M. D. (2002). The interaction of Plasmodiophora brassicae and Arabidopsis thaliana: parameters for disease quantification and screening of mutant lines. Journal of Phytopathology, 150(11-12), 592-605.

Siemens, J., Keller, I., Sarx, J., Kunz, S., Schuller, A., Nagel, W., Schmülling, T., Parniske, M., \& Ludwig-Müller, J. (2006). Transcriptome analysis of Arabidopsis clubroots and disease resistance of cytokinin oxidase/dehydrogenase gene overexpressing plants indicate a key role for cytokinin in disease development. Molecular Plant-Microbe Interactions, 19, 480-494.

Smyth, G. K. (2005). limma: Linear models for microarray data. In R. Gentleman, V. Carey, W. Huber, R. Irizarry, \& S. Dudoit (Eds.), Bioinformatics and computational biology solutions using $R$ and bioconductor (pp. 397-420, Statistics for Biology and Health). New York: Springer.

Svačinová, J., Novák, O., Plačková, L., Lenobel, R., Holík, J., Strnad, M., \& Doležal, K. (2012). A new approach for cytokinin isolation from Arabidopsis tissues using miniaturized purification: pipette tip solid-phase extraction. Plant Methods, 8(1), 1-14.

Tamura, K., Stecher, G., Peterson, D., Filipski, A., \& Kumar, S. (2013). MEGA6: Molecular evolutionary genetics analysis version 6.0. Molecular biology and evolution.

Usadel, B., Poree, F., Nagel, A., Lohse, M., Czedik-Eysenberg, A., \& Stitt, M. (2009). A guide to using MapMan to visualize and compare Omics data in plants: a case study in the crop species, Maize. Plant, Cell \& Environment, 32(9), 12111229.

Vankova, R. (1999). Cytokinin glycoconjugates - distribution, metabolism and function. In M. Strnad, P. Pec, \& E. Beck (Eds.), Advances in regulation of plant growth and development (pp. 67-78). Peres Publishers. 